\title{
Correction to: SpectralTAD: an R package for defining a hierarchy of topologically associated domains using spectral clustering
}

\author{
Kellen G. Cresswell ${ }^{\dagger}$, John C. Stansfield and Mikhail G. Dozmorov ${ }^{*+}$
}

\author{
The original article can be found \\ online at https://doi.org/10.1186/ \\ s12859-020-03652-w. \\ * Correspondence: mikhail. \\ dozmorov@vcuhealth.org \\ ${ }^{\dagger}$ Kellen G. Cresswell and Mikhail G. \\ Dozmorov contributed equally to \\ this work. \\ Department of Biostatistics, Virginia \\ Commonwealth University, \\ Richmond, VA, USA
}

Correction to: BMC Bioinformatics 21, 319 (2020)

$$
\text { https://doi.org/10.1186/s12859-020-03652-w }
$$

Following publication of the original article [1], the authors identified misformatted equations in the published article. The correctly formatted equations are given below.

1. Calculating the normalized symmetric Laplacian:

$$
\bar{L}=D^{-\frac{1}{2}} C D^{-\frac{1}{2}}
$$

2. Solve the generalized eigenvalue problem:

$$
\bar{L} \bar{V}=\lambda \bar{V}
$$

3. The result is a matrix of eigenvectors $\bar{V}_{w \times k}$, where $w$ is the window size, and $k$ is the number of eigenvectors used, and a vector of eigenvalues where each entry $\lambda_{i}$ corresponds to the $i_{t h}$ eigenvalue of the normalized Laplacian $\bar{L}$.

4. Normalize rows and columns to sum to 1 :

$$
\widehat{V_{i .}}=\frac{\bar{V}_{i .}}{\left\|\bar{V}_{i .}\right\|}
$$

5. Find the mean silhouette score over all possible numbers of clusters $m$ and organize into a vector of means: 


$$
\bar{s}_{m}=\frac{\sum_{i=1}^{m} s_{i}}{m}
$$

6. Find the value of $m$ which maximizes $\bar{s}_{m}$

The original article has been updated.

Published online: 27 August 2020

\section{Reference}

1. Cresswell, et al. SpectralTAD: an R package for defining a hierarchy of topologically associated domains using spectral clustering. BMC Bioinformatics. 2020;21:319. https://doi.org/10.1186/s12859-020-03652-w. 